
\section{$E_{\text {citus }}$}

${ }^{1}$ Divison of Respiratory and Critical Care Medicine, University Medicine Cluster, National University Health System, Singapore, Singapore ${ }^{2}$ Department of Medicine, Yong Loo Lin School of Medicine, National University of Singapore, Singapore, Singapore

${ }^{3}$ Department of Pathology, National University Health System, Singapore, Singapore

\section{Correspondence to}

Dr Hiang-Ping Chan, Division of Respiratory and Critical Care Medicine, University Medicine Cluster, National University Health System, 1E Kent Ridge Rd, Singapore 119228, Singapore: chp.chan@gmail.com

\section{Received 3 June 2016} Revised 8 July 2016 Accepted 3 August 2016 Published Online First 31 August 2016

\title{
Use of cryobiopsy during pleuroscopy for diagnosis of sarcomatoid malignant mesothelioma
}

\author{
Hiang-Ping Chan, ${ }^{1,2}$ Mei-Fong Liew, ${ }^{1,2}$ Ju-Ee Seet, ${ }^{3}$ Pyng Lee ${ }^{1,2}$
}

A 74-year-old man presented with cough of 3 weeks' duration. Chest X-ray showed a large left-sided pleural effusion (figure 1) that was a lymphocytic exudate with normal adenosine deaminase level, and negative bacterial and mycobacterial cultures. Flexi-rigid pleuroscopy (LTF 160, Olympus, Japan) revealed candle-wax fibrotic parietal pleura (figure 2), which was sampled using the flexible forceps (FB-55CD-1, Olympus, Japan) with cusps measuring $2.4 \mathrm{~mm}$ when fully open, and a cryoprobe of $2.4 \mathrm{~mm}$

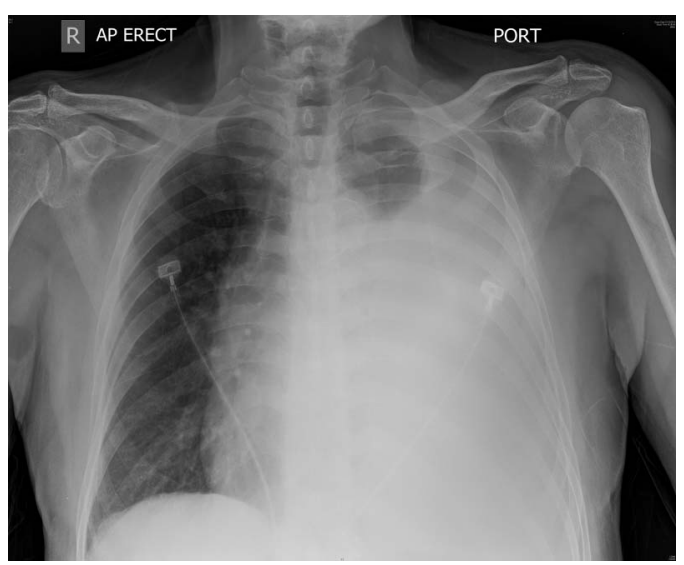

Figure 1 Left large effusion with normal mediastinum. in diameter (20416-032, Erbokryo CA, Erbe, Germany). The cryoprobe was directed to the target area and 4-second freeze time was applied. The pleuroscope and cryoprobe with the pleural specimen attached to the frozen tip were removed en bloc through the trocar. The tissue was retrieved by thawing in normal saline. Cryobiopsies revealed sarcomatoid malignant mesothelioma while those obtained with forceps were non-diagnostic (figure 3).

Diagnosis of mesothelioma can be challenging for early or sarcomatoid subtype as sampling site, technique and instrument can lead to false-negative histology, especially if biopsy samples are of inadequate depth. This arises from the need to fit the forceps through a small working channel of the pleuroscope, thereby limiting the size of the forceps and its mechanical strength in obtaining deep biopsies. Physicians advocate minithoracotomy, video-assisted thoracoscopic surgery (VATS) or insulated-tip knife to procure pleural specimens, which confer diagnostic accuracy ranging from 74$100 \% .^{1-3}$ Grossebner et $a l^{1}$ demonstrated that VATS biopsy achieved 100\% histology diagnosis but led to increased hospitalisation stay due to prolonged air leak. Kao et $a l^{2}$ reported that thoracotomy was superior over VATS in achieving accurate subtype classification ( $83 \%$ vs $74 \%$ ). Nonetheless, flex-rigid pleuroscopy performed under local

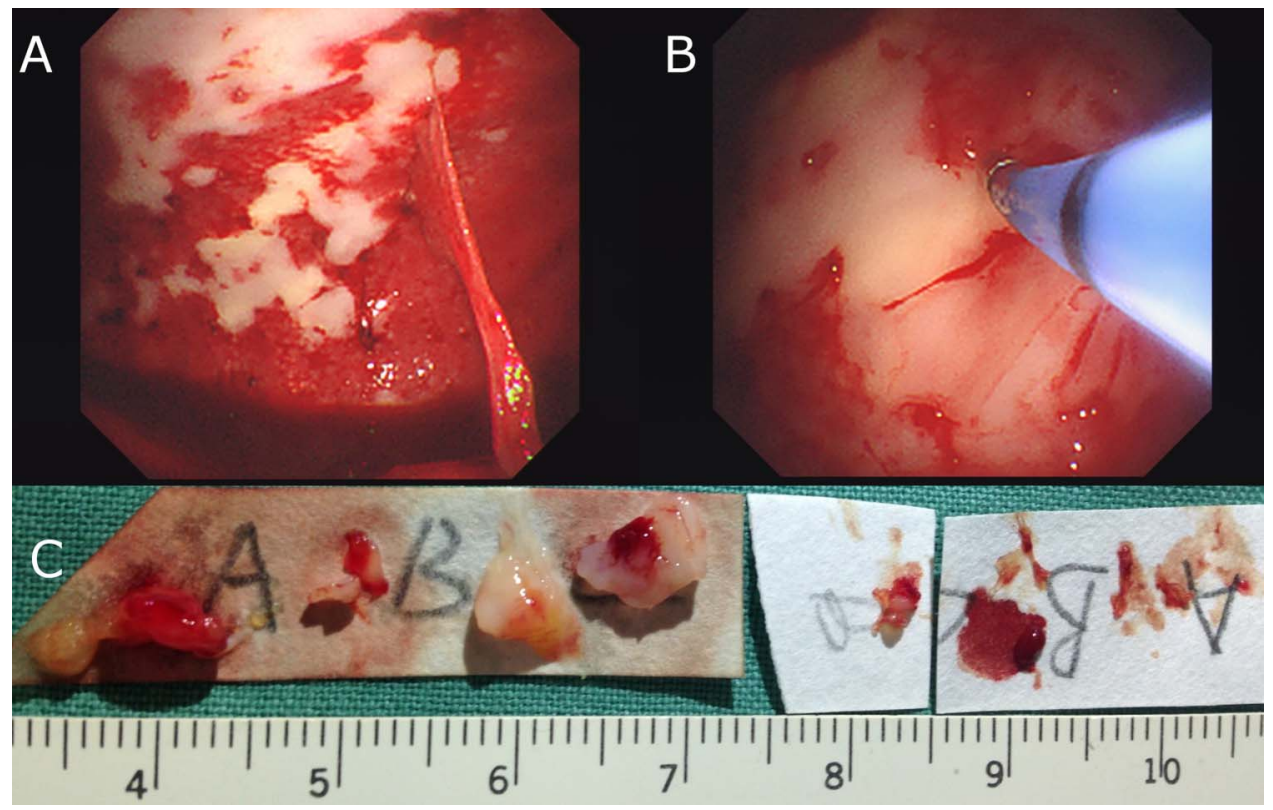

Figure 2 (A) Candle wax parietal pleural abnormalities; (B) cryoprobe biopsy of target area; (C) size of pleural biopsies (left: cryoprobe; right: forceps). 


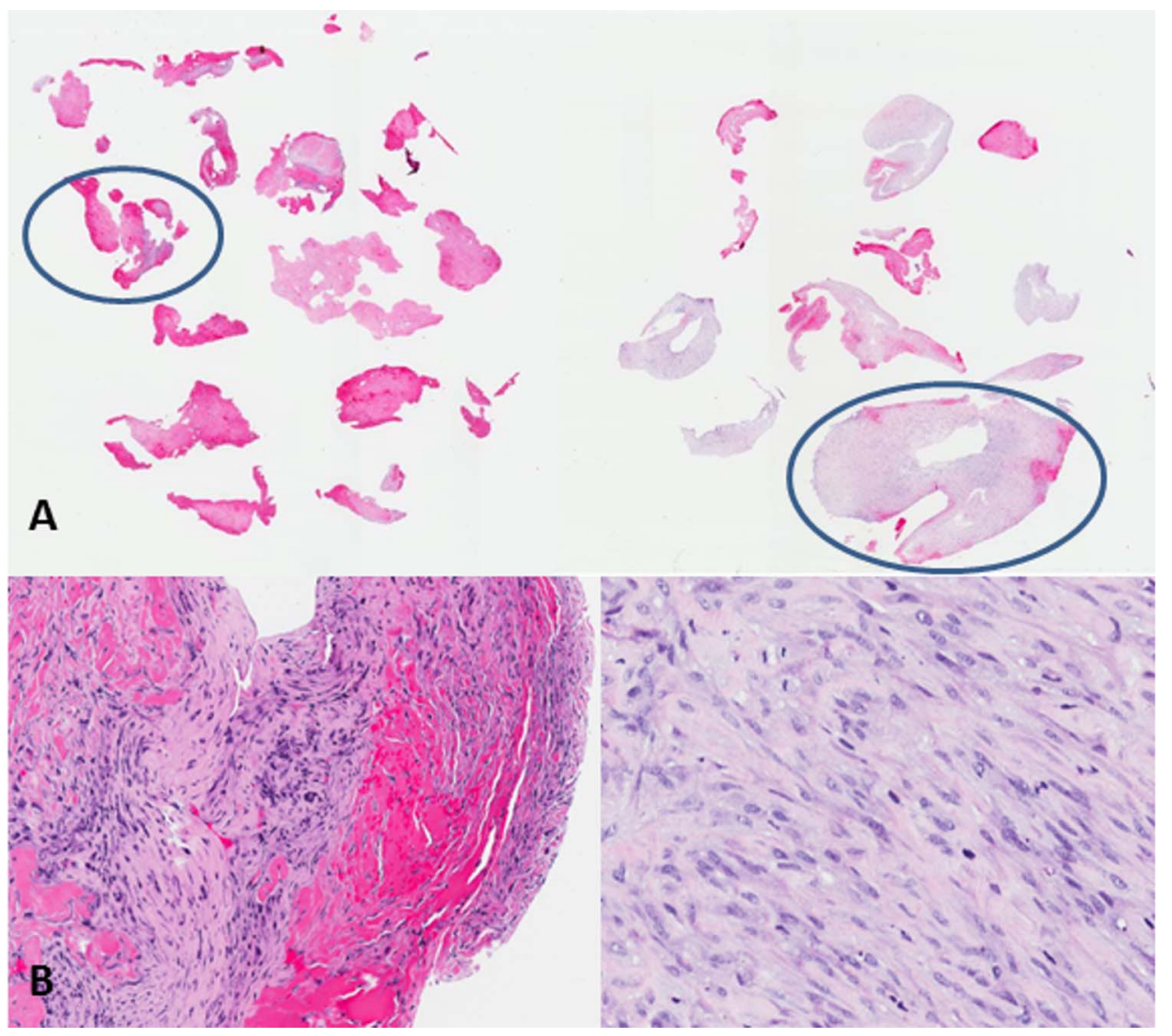

Figure 3 (A) Pleuroscopic forceps biopsies (left) and cryobiopsies (right). Forceps specimens demonstrated predominantly pink-staining surface exudate while thickened pleura was more clearly appreciated in cryobiopsies. (B) Pink-staining fibrin and layered reactive fibroblasts were observed in forceps specimen while cryobiopsy harboured malignant spindle cell tumour ( $\times 20$ original magnification of specimens circled in A).

Table 1 Comparison of studies looking at the use of thoracoscopic cryobiopsy of the pleura

\begin{tabular}{|c|c|c|}
\hline & Thomas et $a l^{5}$ & Rozman et $a l^{4}$ \\
\hline Number of patients & 22 & 15 \\
\hline Gender (male/female) & $21 / 1$ & $12 / 3$ \\
\hline Technique & $\begin{array}{l}2.4 \mathrm{~mm} \text { cryoprobe applied to abnormal parietal pleura } \\
\text { with } 3 \mathrm{~s} \text { freeze time }\end{array}$ & $\begin{array}{l}2.4 \mathrm{~mm} \text { cryoprobe applied to abnormal parietal } \\
\text { pleura with } 3 \mathrm{~s} \text { freeze time }\end{array}$ \\
\hline Size (median, 25-75 IQR) & $10,7-15.8 \mathrm{~mm}$ & - \\
\hline Crush artefacts & $2 / 22(9.1 \%)$ & $18 / 42$ specimens $(42.9 \%)$ \\
\hline Diagnosis & $21 / 22(95.5 \%)$ & $14 / 15(93.3 \%)$ \\
\hline Malignancy & $\begin{array}{l}13 \\
\text { Mesothelioma: } 11 \text { (epithelioid/not specified) } \\
\text { Metastatic cancer: } 1 \\
\text { Lymphoma: } 1\end{array}$ & $\begin{array}{l}14 \\
\text { Mesothelioma: } 9 \\
\text { (not specified) } \\
\text { Adenocarcinoma: } 4 \\
\text { Synovial sarcoma: } 1\end{array}$ \\
\hline Benign fibrinous pleuritis & 8 & 0 \\
\hline Non-specific & 1 & 1 \\
\hline Complications & 5/22 (mild bleeding) & $\begin{array}{l}\text { 14/15 (mild bleeding) } \\
\text { 2/15 (pain) }\end{array}$ \\
\hline
\end{tabular}

anaesthesia remains an attractive diagnostic modality and two small studies have demonstrated excellent safety in obtaining pleural specimens using the flexible cryoprobe (table 1). ${ }^{45}$

Our report describes sarcomatoid malignant mesothelioma diagnosed by pleuroscopic cryobiopsy where corresponding specimens with standard forceps were negative. The cryobiopsies were larger and of sufficient depth with better-preserved architecture compared with those obtained by the flexible forceps. While cryobiopsy is not routinely performed during pleuroscopy, this case highlights its potential role when smooth, fibrotic pleura is encountered.

Contributors $\mathrm{H}-\mathrm{PC}$ and PL were involved in the pleuroscopic biopsy and writing up of the manuscript. M-FL was involved with the radiology and the writing up of the manuscript. J-ES was involved in analysis of the histology and writing up of the manuscript.

Competing interests None declared.

Patient consent Obtained. 
Provenance and peer review Not commissioned; externally peer reviewed.

\section{REFERENCES}

1 Grossebner MW, Arifi AA, Goddard M, et al. Mesothelioma-VATS biopsy and lung mobilization improves diagnosis and palliation. Eur J Cardiothorac Surg 1999;16:619-23.

2 Kao SC, Yan TD, Lee K, et al. Accuracy of diagnostic biopsy for the histological subtype of malignant pleural mesothelioma. J Thorac Oncol 2011;6:602-5.
3 Sasada S, Kawahara K, Kusunoki Y, et al. A new electrocautery pleural biopsy technique using an insulated-tip diathermic knife during semirigid pleuroscopy. Surg Endosc 2009;23:1901-7.

4 Rozman A, Camlek L, Marc Malovrh M, et al. Feasibility and safety of parietal pleural cryobiopsy during semi-rigid thoracoscopy. Clin Respir J 2016;10: 574-8.

5 Thomas R, Karunarathne S, Jennings B, et al. Pleuroscopic cryoprobe biopsies of the pleura: a feasibility and safety study. Respirology 2015;20: 327-32. 